\title{
The Use of Symmetry Chords for Expressing Grey Level Constraints
}

\author{
D. R. Bailes and C. J. Taylor \\ Dept. of Medical Biophysics, Manchester University, \\ Stopford Building, Oxford Road, \\ Manchester M13 9PT.
}

\begin{abstract}
In many object recognition systems only geometric constraints on the boundaries of the objects are used. Many authors have shown the merit in determining the distinctiveness of boundary constraints, and using these to speed up and improve the robustness of object recognition algorithms. However, if the objects have variable shapes or the image is cluttered, then the use of boundary constraints alone can cause object recognition to be be both slow and lacking in robustness. We present a method for expressing grey level constraints using the grey levels along symmetry chords. We describe a method for forming groups of symmetry chords, and calculating their distinctiveness on the basis of the grey levels along the chords in training images. We show how distinct groups of symmetry chords can be used in object recognition by creating evidence images for specific groups of boundary points. Our initial results are promising.
\end{abstract}

\section{Introduction}

Most object recognition methods for $2 \mathrm{D}$ objects fall into the following framework. The object is modelled by its boundary, which is divided into segments, and the object is found by finding part or whole boundary segments. Geometric constraints are used for the matching of image segments to model segments, and to ensure consistency between pairs of matched image and boundary segments. Many authors have shown that using the distinctiveness of a segment or a group of segments can speed up and improve the robustness of an object recognition algorithm [ 1, 2, 3 ].

However, if the objects have variable shapes or the image is cluttered, then the use of boundary constraints alone can cause object recognition to be both slow, and lacking in robustness. The following example illustrates the problem. Two image boundary segments may match two model boundary segments which belong to the same model, and the pair of matches may be geometrically consistent, but the image boundary segments may in fact belong to different objects. These bad matches can significantly reduce the speed and robustness of object recognition. The additional constraint of the expected grey levels between these segments can often exclude such bad matches.

We are interested in finding ways of expressing grey level constraints which satisfy the following criteria:

1. They must model variability, which should be learnt from training data.

2. Be able to cope to some extent with partial occlusion.

3. Be unaffected by scaling the grey levels in the image.

4. Be able to cope with variations in spatial size.

5. Allow the calculation of distinctiveness from training data.

6 . Be reasonably general. 


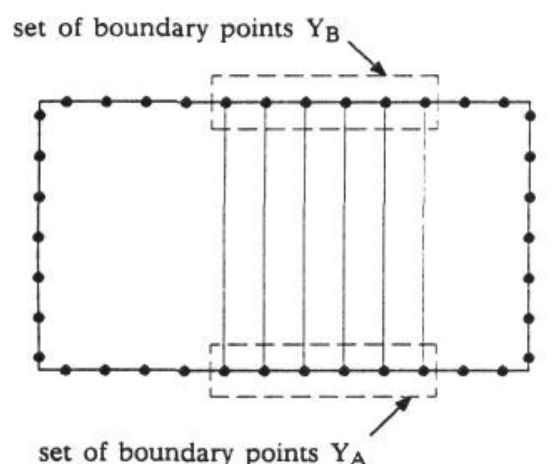

Figure 1. A rectangle and a group of symmetry chords. $Y_{A}$ and $Y_{B}$ are the sets of boundary points which correspond to the possible positions of the two ends of the symmetry chords in this group.

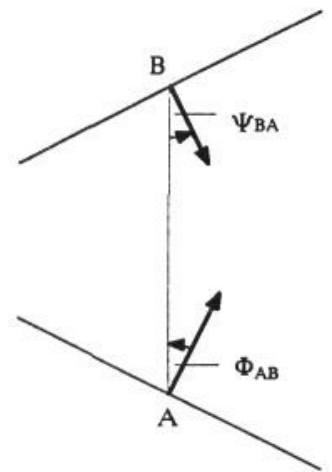

Figure 2. Definition of a symmetry chord.

We have explored the possibility of using the grey level profiles along symmetry chords as grey level constraints, and will give some justification for this in section 2. In many cases, symmetry chords belonging to the same symmetry region have similar grey level profiles, and so these are grouped together to form groups of symmetry chords on the basis of the grey levels along the chords in a set of training images. We then calculate the distinctiveness of each group of chords with respect to all the other groups of chords.

A distinct group of symmetry chords $G$ can be used for object recognition by creating evidence images for the two sets of boundary points $Y_{A}$ and $Y_{B}$ which correspond to the possible positions of the two ends of the symmetry chords in $\mathrm{G}$ ( Fig 1 ). The edge points in the image are found using the method of Canny[ 4 ]. All the possible symmetry chords between pairs of edge points which have a length which lies within the range of lengths of the chords in $\mathrm{G}$ are found. For each chord, the probability that it belongs to $\mathrm{G}$ is calculated using the grey levels along the chord. Using these probabilities, we calculate for each edge point, the probability that it belongs to the set of boundary points $\mathrm{Y}_{\mathrm{A}}$, and the probability that it belongs to the set of boundary points $Y_{B}$, thus creating two probability images. These probability images contain far fewer unwanted points than the original edge image, and can easily be incorporated into any of the standard object recognition methods.

\section{Grey levels along symmetry chords}

In this section we will explain why we have chosen the grey level profiles along symmetry chords as a way of expressing grey level constraints. We will also describe how the grey levels are represented.

We are trying to find distinctive constraints which apply to parts of the grey level landscape. There are a number of options as to what kind of parts of the landscape to use: $2 \mathrm{~d}$ patches of any shape; curves of any shape; or chords. The advantage of using $2 \mathrm{~d}$ patches rather than curves or chords is that there is a better chance of finding patches which are very distinctive, since they contain more infor- 
mation. However, the extremely large number of possible patches makes it difficult to construct an automatic method for finding distinctive constraints. There are also a large number of possible curves, and therefore we have decided to try to find distinctive chords.

We will consider only the chords which begin and end on object boundaries. This has the advantage that it greatly reduces the number of possible chords. One option would be to use all such chords, and parameterise each chord by the following parameters: length; the angles between the boundary normals and the chord; and the grey levels along the chord. However, the number of possible chords is still very large. If we model the boundaries in an image by $\mathrm{N}$ discrete points, then there are $\mathrm{O}\left(\mathrm{N}^{2}\right)$ possible chords. For each of these chords we would have to collect the grey level profiles from several training images. $\mathrm{N}$ does not have to be too large before this results in an unmanageable amount of data which has to be stored and processed.

The number of of chords can be reduced by putting a constraint on the angles between the chord and the boundary normals. We have investigated the constraint that a chord must be a symmetry chord [ 5, 6 ]. In Fig $2 \Phi_{A B}$ is the angle of the symmetry chord $\mathrm{AB}$ with respect to the boundary normal at $\mathrm{A}$, and $\Psi_{\mathrm{BA}}$ is the angle of the boundary normal at point $\mathrm{B}$ with respect to the chord $\mathrm{BA}$. The chord $\mathrm{AB}$ is a symmetry chord if $\Phi_{\mathrm{AB}}=\Psi_{\mathrm{BA}}$.

The locus of the midpoint of the symmetry chords of a boundary takes the form of a number of disconnected symmetry axes. The set of chords associated with a symmetry axis can be considered to 'cover' a region, and we refer to such a region as a symmetry region. For many objects, symmetry chords in the same symmetry region have similar grey level profiles. Thus we are unlikely to be able to find a symmetry chord which is distinct with respect to all other chords. Rather, we hope to find groups of chords which are distinct with respect to all other groups. By moving from trying to find a distinct chord to finding a distinct group we are reducing the specificity of the method, but we are increasing its robustness to occlusion. A symmetry region can be partially occluded, and still have some of its symmetry chords unoccluded.

We have investigated representing the grey levels along a symmetry chord in four different ways: either the grey levels or the values of the component of the gradient in the direction of the chord are used; in each case either the unnormalised values are used, or the values are normalised by dividing the values by the sum of the absolute values along the chord. The values of the component of the gradient in the direction of a chord are obtained from the $\mathrm{x}$ and $\mathrm{y}$ components of the gradient of the Gaussian smoothed images which are produced by the first stage of the Canny edge finding scheme. To ensure a fair comparison, the values of the grey values are taken from images which have been smoothed by a Gaussian filter with the same standard deviation as was used for the edge detection. If it is required that the constraints be unaffected by scaling the grey levels in the image, then the values must be normalised. The advantage of using the component of the gradient in the direction of the chord is that the constraints are also unaffected by a uniform constant being added to the grey levels of the image.

For convenience we will often refer to the values of any of these representations of the grey levels along a chord as 'the grey levels along the chord', and denote their values by the vector $\mathbf{g}$. The grey levels along a chord are not independent, and so we 


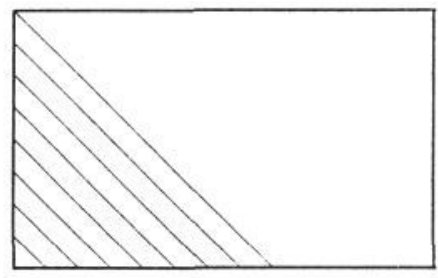

Figure 3. An example of an unwanted symmetry region.

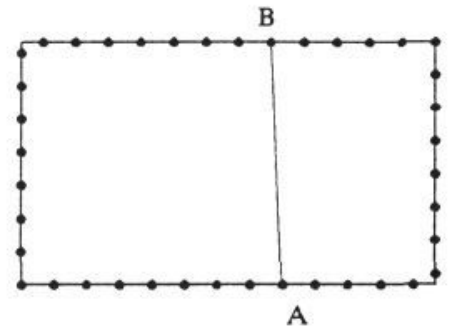

Figure 4. A symmetry chord for a discrete boundary.

describe the distribution of the grey levels by their mean $\overline{\mathbf{g}}$, and their covariance matrix $\mathbf{S}$.

\section{Finding distinct groups of chords from training data}

We now describe an automatic method for finding distinct groups of chords. For each symmetry region we need to end up with a discrete set of chords, so that the grey levels along each chord can be sampled from the training images. We have therefore decided to model the object boundary as a discrete set of points, and to calculate the symmetry chords only between these points. Initially, we will only consider boundaries which are slightly variable.

\subsection{Calculating the symmetry chords}

We do not want to calculate all the symmetry chords for two reasons. Firstly, the set of chords in the symmetry region of the rectangle shown in Fig 3 have widely varying lengths and are unlikely to have similar grey level profiles. We are therefore not interested in such symmetry regions. Secondly, there is the well known problem that all the chords of a circle are symmetry chords. We have decided to find only the chords which are diameters.

In the continuous case, the condition for a symmetry chord is that $\Phi_{\mathrm{AB}}=\Psi_{\mathrm{BA}}$ exactly. However, in the discrete case, this has to be relaxed. Consider point $\mathrm{A}$ in Fig 4 which lies on the boundary of a rectangle. In general, there will not be a point on the opposite side of the rectangle which lies directly opposite point $A$. The condition for a symmetry chord has to become $\left|\Phi_{A B}-\Psi_{B A}\right|<t_{1}$, where $t_{1}$ depends on the spacing of the points along the boundary. There may be more than one point opposite point $A$ which satisfies our relaxed condition, and therefore we choose the chord for which $\left|\Phi_{\mathrm{AB}}-\Psi_{\mathrm{BA}}\right|$ is a minimum with respect to $\mathrm{B}$.

By introducing a second condition that $\left|\Phi_{A B}\right|+\left|\Psi_{B A}\right|$ is a local minimum with respect to $\mathrm{B}$, we find only the symmetry chords in circles which are diameters. $\mathrm{A}$ third condition that $\left|\Phi_{\mathrm{AB}}\right|<\mathrm{t}_{2}$ rules out symmetry regions like the example in Fig 3. Thus our set of conditions for a symmetry chord are:

$$
\begin{aligned}
& \left|\Phi_{A B}-\Psi_{B A}\right|<t_{1}, \\
& \left|\Phi_{A B}\right|<t_{2}, \\
& \left|\Phi_{A B}-\Psi_{B A}\right|+\left|\Phi_{A B}\right|+\left|\Psi_{B A}\right| \text { is a local minimum with respect to } B .
\end{aligned}
$$

The third condition is valid for both axial and circular regions. 


\subsection{Grouping the chords into symmetry region chord sets}

We refer to the set of chords which belong to a symmetry region as a symmetry region chord set. For the symmetry chords of a discrete boundary, we define a symmetry region chord set as follows. Each chord is specified by its start point a, and its end point $b$, where $a=1 \ldots \mathrm{N}_{\text {bound }}$, and $\mathrm{b}=1 . . \mathrm{N}_{\text {bound. }}$. A symmetry region chord set is an ordered set of symmetry chords $\left(a_{j}, b_{j}\right), j=1 \ldots N_{\text {region }}$, such that:

1. $a_{\mathrm{j}}$ is monotonically increasing.

$b_{j}$ is monotonically increasing or decreasing.

2. $a_{j}-a_{j-1}<=1$

$$
\left|b_{j}-b_{j-1}\right|<=1
$$

\subsection{Getting grey level data from the training images}

For each image in the training set, the model boundary is matched to the image manually, and for each of the symmetry chords the grey levels are sampled along the chord. For a given symmetry chord we use the same number of equally spaced sampling points for each image. This ensures that the method can cope with variations in spatial size. The number of sampling points is determined by the maximum length of the chord in the training images, and the standard deviation of the Gaussian filter used to smooth the images. We currently sub-sample by a factor of 2 times the standard deviation. We compute the mean $\overline{\mathbf{g}}$, and the covariance matrix $\mathbf{S}$ for each chord.

\subsection{Forming groups of similar chords}

We currently choose groups of similar chords manually. However we envisage an automatic method of grouping which consists of two stages: for each symmetry region we form groups of chords, such that within groups there is little variation in $\overline{\mathbf{g}}$ and $\mathbf{S}$; then, within an object, similar groups are merged. For each group, the overall values of $\overline{\mathbf{g}}$ and $\mathbf{S}$ are calculated, and these values are used to calculate how distinct each group is with respect to all the other groups. Thus each group has the following parameters:

1. A measure of distinctiveness.

2. Overall values of $\overline{\mathbf{g}}$ and $\mathbf{S}$.

3. The range of angles of the symmetry chords with respect to the boundary normal at $\mathrm{A}, \Phi_{A B_{1}}$ to $\Phi_{A B_{2}}$.

4. The range of lengths of the chords, $1_{\min }$ to $l_{\max }$.

5. The number of sampling points for the chord $n_{s}$.

6. The set of boundary points $Y_{A}$ which corresponds to the possible positions of point $\mathrm{A}$.

7. The set of boundary points $Y_{B}$ which corresponds to the possible positions of point $\mathrm{B}$. 
Figure 5. Finding the edge points which form symmetry chords with the point $\mathbf{x}_{1}$.

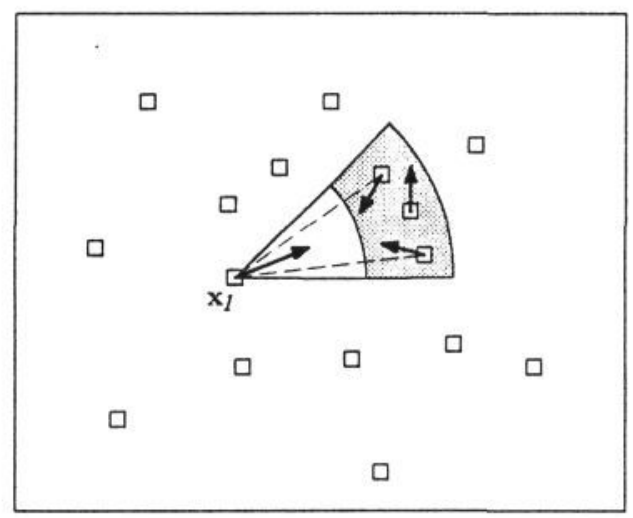

\section{An application of grey level constraints}

If an object contains one or more distinct groups of chords, then we can usefully create evidence images for specific groups of boundary points. Given an image in which we want to find the object, the edge points in the image are found using Canny's method, except that we do not apply any thresholding. For a given group of symmetry chords $G_{i}$, we find all the possible symmetry chords between pairs of edge points which have a length in the range $l_{\min }$ to $l_{\max }$, and an angle with respect to the boundary normal at A in the range $\Phi_{A B_{1}}$ to $\Phi_{A B_{2}}$. For each symmetry chord, the probability that it belongs to $G_{j}$ is calculated using the grey levels along the chord, which are sampled at the same number of equally spaced points for each chord. Using these probabilities, we calculate for each edge point, the probability that it belongs to the set of boundary points $\mathrm{Y}_{\mathrm{A}_{\mathrm{i}}}$, and the probability that it belongs to $Y_{B_{i}}$, thus creating two probability images.

The probabilities that an edge point belongs to $Y_{A_{i}}$, or $Y_{B_{i}}$ are calculated as follows. If a point $x_{1}$ is hypothesised as a member of $Y_{A_{i}}$, then the other end of the symmetry chord must lie within the sector of an annulus which has radii $l_{\min }$ and $l_{\max }$, and range of angles $\Theta_{A}+\Phi_{A B_{1}}$ to $\Theta_{A}+\Phi_{A B_{2}}$, where $\Theta_{\mathrm{A}}$ is the angle of the gradient at $\mathbf{x}_{1}$. In general, there will be a number of edge points within this sector, and of these, a number will form symmetry chords with point $\mathbf{x}_{1}$, as shown in Fig 5 . Let set $X_{B}$ be the set of edge points within the sector which form a symmetry chord with point $x_{1}$. The point $x_{1}$ is a member of $Y_{A_{i}}$ and the point $x_{2}$ is a member of $Y_{B_{i}}$ if, and only if the chord $\left(x_{1}, x_{2}\right)$ belongs to the chosen group $G_{i}$ on the basis of its grey level profile. Therefore the point $\mathrm{x}_{2}$ in set $\mathrm{X}_{\mathrm{B}}$ which is most likely to belong to $Y_{B_{1}}$ is the point for which the probability that the chord $\left(x_{1}, x_{2}\right)$ belongs to the group of chords is a maximum. Hence the probability that point $\mathbf{x}_{1}$ is a member of $\mathrm{Y}_{\mathrm{A}_{\mathrm{i}}}$ is given by:

And similarly:

$$
P\left(\mathbf{x}_{1} \text { is a member of } Y_{A_{i}} \mid \mathbf{x}_{1}, X_{B}\right)=\max _{\mathbf{x}_{2} \in X_{B}} P\left(G_{i} \mid \mathbf{g}\left(\mathbf{x}_{1}, \mathbf{x}_{2}\right)\right)
$$

$$
P\left(\mathbf{x}_{2} \text { is a member of } Y_{B_{i}} \mid \mathbf{x}_{2}, X_{A}\right)=\max _{\mathbf{x}_{1} \in X_{A}} P\left(G_{i} \mid \mathbf{g}\left(\mathbf{x}_{1}, \mathbf{x}_{2}\right)\right)
$$




\subsection{Probabilities}

It can be seen that we need to calculate the probability that a particular chord comes from the chosen group of chords. Using Bayes theorem:

$$
\begin{aligned}
P\left(G_{i} \mid \mathbf{g}\right) & =\frac{P\left(G_{i}\right) P\left(\mathbf{g} \mid G_{i}\right)}{P\left(G_{i}\right) P\left(\mathbf{g} \mid G_{i}\right)+P\left(G_{i}^{c}\right) P\left(\mathbf{g} \mid G_{i}^{C}\right)} \\
& =\frac{p_{i} f_{i}(\mathbf{g})}{p_{i} f_{i}(\mathbf{g})+p_{c} f_{C}(\mathbf{g})}
\end{aligned}
$$

where $G_{i}^{C}$ is the complement of $G_{i}, p_{i}$ and $p_{C}$ are the prior probabilities that a chord belongs to $G_{i}$ and $G_{i}^{C}$ respectively, and $f_{i}(g)$ and $f_{C}(g)$ are the probability densities of $G_{i}$ and $G_{i}^{C}$ respectively. The main decision is what form the distribution for $f_{C}(g)$ should take. We have made the assumption that this is a normal distribution, even though we know that there will be spikes in the distribution due to other distinct groups of chords.

It is computationally efficient to compute the probability via the odds ratio:

$$
\begin{aligned}
& P\left(G_{i} \mid \mathbf{g}\right)=\frac{\text { odds }}{1+\text { odds }} \\
& \text { odds }=\frac{P\left(G_{i} \mid \mathbf{g}\right)}{P\left(G_{i}^{C} \mid \mathbf{g}\right)}=\frac{p_{i} f_{i}(\mathbf{g})}{p_{c} f_{c}(\mathbf{g})} \\
& =\frac{\frac{p_{i}}{(2 \pi)^{\frac{n_{s}}{2}}\left|\mathbf{S}_{i}\right|^{\frac{1}{2}}} \exp \left(-\left(\mathbf{g}-\overline{\mathbf{g}}_{i}\right)^{\prime} \mathbf{S}_{i}^{-1}\left(\mathbf{g}-\overline{\mathbf{g}}_{i}\right) / 2\right)}{\frac{p_{c}}{(2 \pi)^{\frac{n_{s}}{2}}\left|\mathbf{S}_{c}\right|^{\frac{1}{2}}} \exp \left(-\left(\mathbf{g}-\overline{\mathbf{g}}_{C}\right)^{\prime} \mathbf{S}_{c}^{-1}\left(\mathbf{g}-\overline{\mathbf{g}}_{C}\right) / 2\right)} \\
& =\left(\frac{p_{i}}{p_{c}}\right)\left(\frac{\left|\mathbf{S}_{C}\right|}{\left|\mathbf{S}_{i}\right|}\right)^{\frac{1}{2}} \exp \left(-\mathbf{g}^{\prime}\left(\mathbf{S}_{i}^{-1}-\mathbf{S}_{C}^{-1}\right) \mathbf{g} / 2+\left(\overline{\mathbf{g}}^{\prime} \mathbf{S}_{i}^{-1}-\overline{\mathbf{g}}_{C}{ }^{\prime} \mathbf{S}_{C}^{-1}\right) \mathbf{g}-\left(\overline{\mathbf{g}}_{i}^{\prime} \mathbf{S}_{i}^{-1} \overline{\mathbf{g}}_{i}-\overline{\mathbf{g}}_{C} \mathbf{S}_{c}^{-1} \overline{\mathbf{g}}_{C}\right) / 2\right)
\end{aligned}
$$

\section{Results}

We have applied the method described above to both printed circuit board ( PCB) and brake assembly images, and have obtained promising results with both sets of images. The method for automatically finding the distinct groups of chords is not complete, and therefore we have chosen groups manually which look as if they are distinct.

We present the results of one of our experiments on PCB images. Both the training set and the validation set contain 8 images, and an example of one of the training images is shown in Fig 6 . We have chosen the group of symmetry chords which lie across the bodies of the resistors (there are 41 and 38 resistors in the training and validation sets respectively ). The edge points which belong to the sets $Y_{A}$ and $Y_{B}$ were manually labelled, and these are shown in Fig $6 \mathrm{c}$. The symmetry chords which were used for collecting training data from this image are shown in Fig 6d. An example of a validation image, its edge magnitude image, and the set of points which were manually labelled as belonging to $Y_{A}$ or $Y_{B}$ are shown in Fig 7 a, 


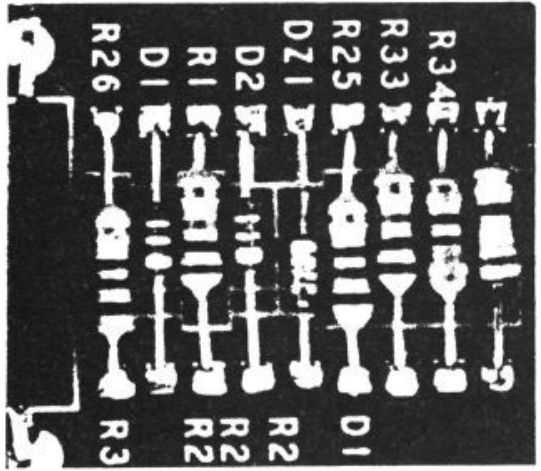

( a )

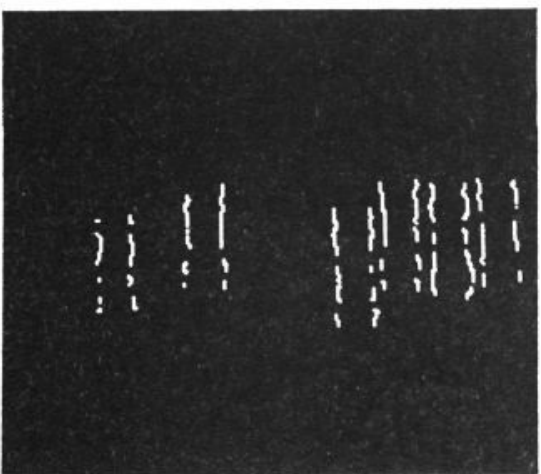

(c)

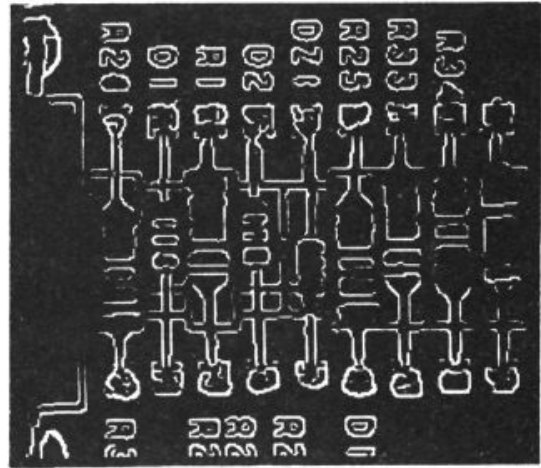

( b )

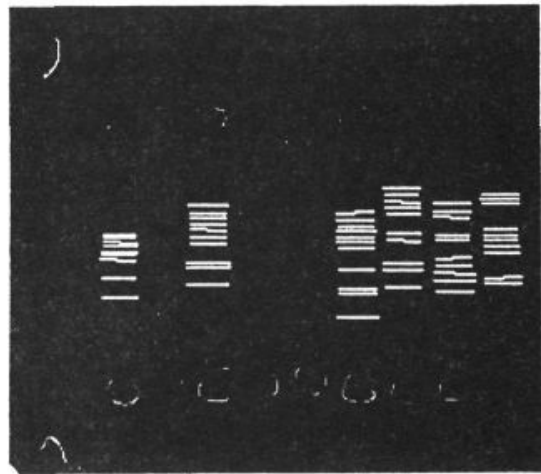

( d )

Figure 6. An example of a training image. a) the original image, b) edge magnitude image, c) edge points which were manually labelled as belonging to sets $Y_{A}$ or $\left.Y_{B}, d\right)$ symmetry chords which were used for collecting the training data.

$\mathrm{b}$ and $\mathrm{c}$ respectively. Fig $7 \mathrm{~d}$ shows the edge points for which the computed probability that they belong to the set $\mathrm{Y}_{\mathrm{A}}$ is greater than 0.5 . Because the grey level profiles along each of the symmetry chords in the chosen group is roughly symmetric about the midpoint of the chord, the probability that a point belongs to the set $Y_{A}$ is roughly the same as the probability that it belongs to the set $\mathrm{Y}_{\mathrm{B}}$. Hence this image corresponds to the membership of the union of the sets $\mathrm{Y}_{\mathrm{A}}$ and $\mathrm{Y}_{\mathrm{B}}$.

The method was quantitatively evaluated by computing how well it classified the edge points in the validation set. If the value of a pixel in a probability map is greater than a threshold, then we consider it to be classified as a member of $\mathrm{Y}_{\mathrm{A}}$ or $\mathrm{Y}_{\mathrm{B}}$ of the chosen group of chords. ROC curves for the four methods of representing the grey levels were computed by varying this threshold, and are shown in Fig 8. The curves are averages over the validation set, and only edge points which satisfied the geometric constraint of being an end of a symmetry chord with length in the range $I_{\min }$ to $I_{\max }$, and angle with respect to the boundary normal at $A$ in the range $\Phi_{A B_{1}}$ to $\Phi_{A B_{2}}$ were classified. The average number of boundary points per image which belonged to the sides of the resistors was 449 , and of these 412 satisfied the geometric constraint; the average number of other boundary points was 12623 , and 


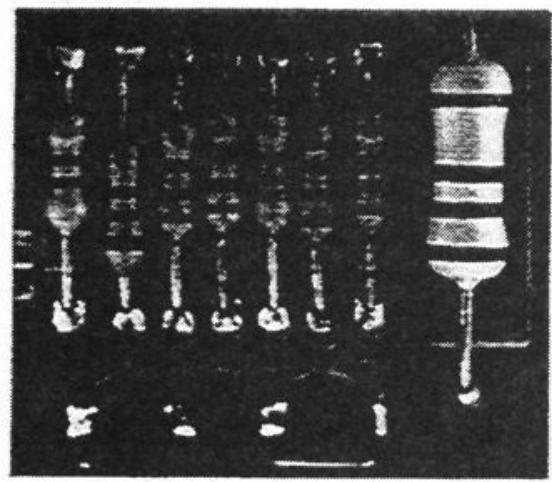

( a )

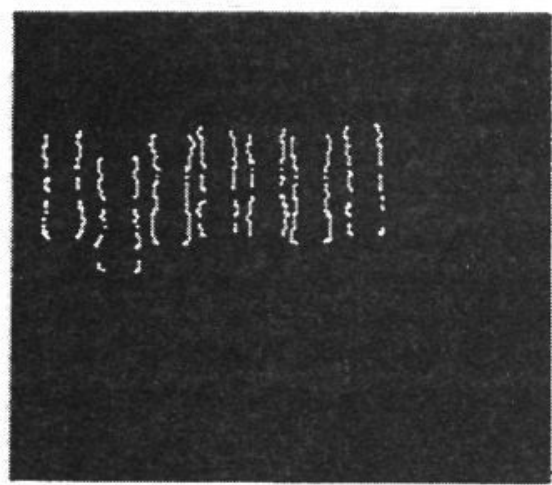

( c)

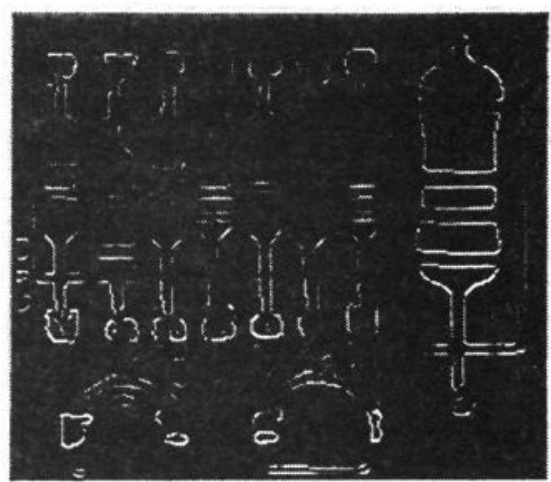

( b )

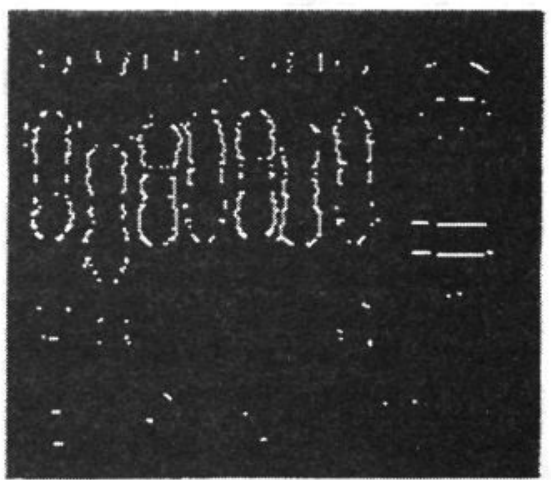

(d)

Figure 7. An example of a validation image. a) original image, b) edge magnitude image, c) edge points which were manually labelled as belonging to $Y_{A}$ or $\left.Y_{B}, d\right)$ edge points for which the computed probability that they belong to $Y_{A}$ is greater then 0.5 .

Figure 8. Classification results: ROC curves for the 4 ways of representing the grey level profiles, and for the geometric constraints alone:
A: grey levels
$B$ : normalised grey levels
$C$ : gradient
$D$ : normalised gradient
E: geometric constraint alone

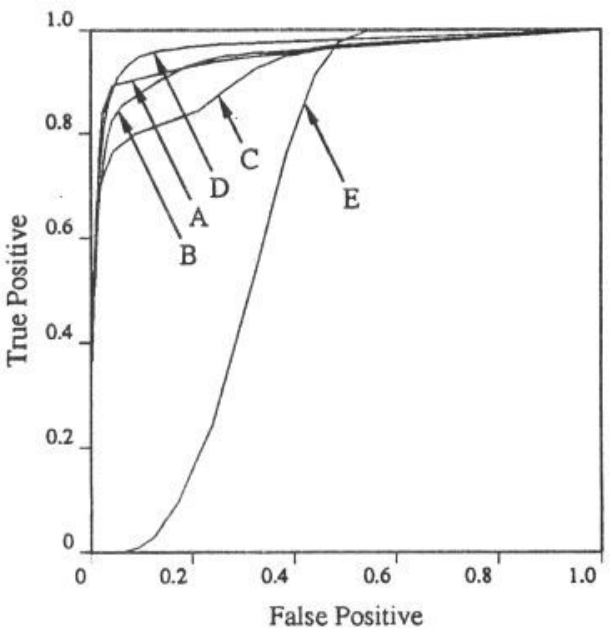


of these 6967 satisfied the geometric constraint. It can be seen that the normalised gradient representation has the best ROC curve, and is the preferred representation. We have also given the classification results for the use of the geometric constraint alone: the ROC curve was obtained by thresholding the values of edge magnitude of the edge points which satisfied the geometric constraint. It can be seen that using the grey level constraint as well as the geometric constraint greatly decreases the number of misclassifications.

For each $256 \times 256$ PCB image the algorithm takes about 17 seconds on a Sun Sparc 2 workstation. For the chosen group of chords: $1_{\min }=14,1_{\max }=20$, $\Phi_{A B_{1}}=-18^{\circ}$, and $\Phi_{A B_{2}}=18^{\circ}$. No prior knowledge of the orientation of the symmetry chords is used.

\section{Conclusions}

We have developed a method of expressing grey level constraints which uses the grey level profiles along symmetry chords. We represent a grey level profile by the normalised values of the component of the gradient in the direction of the chord. These grey level constraints satisfy the criteria given in the introduction: they model the variability in the training data; are able to cope to some extent with partial occlusion; they are unaffected by scaling the grey levels in an image; they are able to cope with variations in spatial size; they allow the calculation of distinctiveness from training data; and are reasonably general. We have outlined an automatic method for grouping the chords, but the implementation of this needs completing.

We have shown how distinct groups of symmetry chords can be used in object recognition by creating evidence images for specific groups of boundary points. These evidence images contain far fewer unwanted points than the original edge image. The results are encouraging.

There are two straightforward generalisations of the method. Firstly, rather than the grey levels along symmetry chords, the values of any intrinsic image, eg texture, could be used. Secondly, instead of the symmetry chords between edge points, the symmetry chords between ridge points or trough points could be used.

\section{Acknowledgements}

Various members of the Wolfson Unit, and especially Dave Cooper, Tim Cootes, and Jim Graham for their helpful comments. This work was funded by SERC project grant no. GR/F63633.

\section{References}

[1] R.C. Bolles, and R.A. Cain, Recognising and locating partially visible objects: the local-feature-focus method. Int. J. Rob. Res. 1982; 1: 57-82.

[2] J.L. Turney, T.N. Mudge, and R.A. Voltz, Recognizing partially occluded parts. IEEE Trans. PAMI 1985; 7: 410-421.

[3] T.N. Mudge, J.L. Turney, and R.A. Voltz, Automatic generation of salient features for the recognition of partially occluded parts. Robotica 1987; 5: 117-127.

[4] J. Canny, A computational approach to edge detection. IEEE Trans PAMI 1986; 8: 679-698.

[5] H. Blum, Biological shape and visual science (part 1). J. Theor. Biol. 1973; 38: 205-287.

[6] J. M. Brady, and H. Asada, Smoothed local symmetries and their implementations. Intern. J. Robot. Res. 1984; 3: 36-61. 